\title{
Recomendaciones para reducir el riesgo de lesiones cutáneas secundarias al uso de elementos de protección personal (EPP) frente a la pandemia por SARS-CoV-2/ COVID-19 para el personal de la salud
}

\author{
Claudia Marcela Arenas Soto; ; Alexander Castañeda Morales²; Claudia Rocío Cáceres \\ Bonilla3; Jorge Espinosa Reyes; Alberto Luis Díaz Díaz; Héctor Camilo Pérez Cely ${ }^{6}$; \\ Adriana Motta Beltrán7; Julio Roberto Amador Patarroyo ${ }^{8}$; Isabel Cristina Cuellar Ríos9; \\ Mauricio Torres Pradilla ${ }^{10}$; Héctor Castellanos Lorduy ${ }^{11}$; Luis Arturo Gamboa Suárez ${ }^{12}$; \\ Claudia Juliana Díaz Gómez ${ }^{13}$; Jairo Victoria Chaparro ${ }^{14}$; Liliana Eugenia Muñoz \\ García $^{15}$; Ángela María Londoño García ${ }^{16}$; Mónica Salazar Soto ${ }^{17}$; Margarita Velásquez \\ Lopera $^{18}$; Hernando Mosquera Sánchez ${ }^{19}$; Francisco Miguel Camacho Chaljub ${ }^{20}$; Felipe \\ Jaramillo Ayerbe ${ }^{21}$
}

\section{RESUMEN}

El virus SARS-CoV-2 causante de la enfermedad COVID-19 es altamente contagioso y debido a que la propagación nosocomial ha sido bien documentada, se han implementado directrices que incluyen el uso de elementos de protección personal (EPP) con el objetivo de minimizar el riesgo de contagio en el personal de la salud. Secundario al uso continuo, repetitivo y prolongado de estos dispositivos, se pueden desencadenar procesos inflamatorios cutáneos tales como: dermatitis de contacto, prurito o urticaria por presión, o exacerbar procesos inflamatorios preexistentes como dermatitis seborreica, acné, entre otros. Por lo anterior, la Asociación Colombiana de Dermatología y Cirugía Dermatológica (AsoColDerma), en alianza con el Hospital Universitario Centro Dermatológico Federico Lleras Acosta, como ente asesor del Gobierno colombiano, nos permitimos hacer las siguientes recomendaciones encaminadas a reducir el riesgo de lesiones cutáneas derivadas del uso de los equipos de protección personal.

Estas recomendaciones son de orientación general para la práctica clínica, y el personal de la salud las debe seguir de forma individualizada, de acuerdo con sus necesidades y con los recursos disponibles.

PALABRAS CLAVE: coronavirus; trabajadores; salud; equipo de protección personal; ropa de protección; detergentes; dermatitis por contacto; úlceras de presión; hiperhidrosis.

Correspondencia: Claudia Marcela Arenas Soto; email: draclaudiaarenas@gmail.com

Recibido: 21/04/20; aceptado: 29/04/20

Cómo citar: Arenas-Soto CM, Castañeda-Morales A, Cáceres-Bonilla CR, Espinosa-Reyes J, Díaz-Díaz AL, Pérez-Cely HC, Motta-Beltrán A, Amador-Patarroyo JR, Cuellar-Ríos IC, Torres-Pradilla M, Castellanos-Lorduy H, et al. Recomendaciones para reducir el riesgo de lesiones cutáneas secundarias al uso de elementos de protección personal (EPP) frente a la pandemia por SARS-CoV-2/COVID-19 para el personal de la salud. Rev Asoc Colomb Dermatol. 2020;28(1):27-40. DOI: https://doi.org/10.29176/2590843X.1488

Financiación: ninguna, conflictos de interés: ninguno 


\section{RECOMMENDATIONS TO REDUCE THE RISK OF SECONDARY SKIN INJURIES TO THE USE OF PERSONAL PROTECTION ELEMENTS (PPE) AGAINST THE SARS-COV-2/COVID-19 PANDEMIC FOR HEALTH WORKERS}

\section{SUMMARY}

The SARS-CoV-2 virus causing the COVID-19 disease is highly contagious and because nosocomial spread has been well documented. Guidelines have been implemented that include the use of personal protection elements (PPE) in order to minimize the risk of contagion in health workers; secondary to the continuous, repetitive and prolonged use of these devices, inflammatory skin processes such as contact dermatitis, pruritus, pressure urticaria or exacerbating pre-existing inflammatory processes such as seborrheic dermatitis, acne, among others, can be triggered. For this reason, the Colombian Association of Dermatology and Dermatological Surgery (AsoColDerma) in alliance with the Hospital Universitario Centro Dermatológico Federico Lleras Acosta, as an advisory organization to the Colombian Government, we allow ourselves to make the following recommendations aimed at reducing the risk of skin lesions derived from use of personal protective equipment.

These recommendations are general guidelines for clinical practice, and must be followed individually by health personnel according to their needs and available resources.

KEY WORDS: Coronavirus; Workers; Health; Personal protective equipment; Protective clothing; Cleansing; Contact dermatitis; Pressure ulcers; Hyperhidrosis.

\section{INTRODUCCIÓN}

A finales del año 2019, se produjo un brote de infección respiratoria grave en Wuhan, capital de la provincia de Hubei, en China, identificándose un betacoronavirus como el agente causal, al cual se le dio el nombre de $S A R S$-CoV-2. Se le consideró como una zoonosis y el 12 de febrero de 2020 la Organización Mundial de la Salud (OMS) la denominó enfermedad por coronavirus 2019 (COVID-19). Rápidamente el virus se diseminó por toda China, y luego por todo el planeta, por lo que fue declarada pandemia por parte de la OMS. Para el 11 de abril de 2020, la enfermedad se encontraba ya en 193 países y regiones, afectaba a 1.700.769 personas y había causado la muerte a 103.141 de ellas (según el conteo de la AFP a partir de balances oficiales).

En Colombia, hasta la fecha, existen 2709 casos reportados, 100 muertos y 214 pacientes recuperados (según datos de la ONU al 11 de abril de 2020).

Además de China, los países con mayor número de casos son Italia, España y Estados Unidos, que ahora es el epicentro de la pandemia. Dada su alta transmisi- bilidad, el número de enfermos rápidamente desborda la capacidad de las clínicas y los hospitales, poniendo en alto riesgo al personal de salud. En España, cerca del $14 \%$ de los trabajadores de la salud fueron infectados, con las consecuencias que esto acarrea, no solo por sobrecargar aún más al sistema sanitario, sino por tratarse de un frente menos para enfrentar la contingencia. Al 5 de abril de 2020, se había informado el fallecimiento de 198 médicos por COVID-19 en todo el mundo, de los cuales, 79 murieron en Italia. El 11 de abril, en Colombia, en la ciudad de Bogotá, fallecieron 2 médicos por COVID-19. Por lo anterior, es de vital importancia que las entidades de salud extremen las medidas para mitigar la transmisión del virus en los ambientes hospitalarios, mediante protocolos estrictos de aislamiento de aerosoles y de contacto. Para esto, es fundamental utilizar elementos de protección personal (EPP); sin embargo, el uso prolongado y repetido de tales elementos también puede ocasionar problemas dermatológicos de distinto orden y gravedad.

Teniendo en cuenta todo lo anterior, en este documento se describen las complicaciones que se presentan con mayor frecuencia por el uso de los EPP y se 
formulan las recomendaciones para la prevención y el tratamiento de estas.

El virus SARS-CoV-2 es un virus ARN de cadena simple, que causa la enfermedad del coronavirus 2019 (COVID19). Este virus, aunque no es tan letal como otros virus que causan el síndrome respiratorio agudo grave (SARS, por sus siglas en inglés), se caracteriza por ser altamente transmisible y mortal en pacientes con factores de riesgo, tales como:

- Edad avanzada (>6o años)

- Enfermedad cardiovascular

- $\quad$ Diabetes mellitus

- Hipertensión arterial

- Enfermedad pulmonar/EPOC

- Cáncer

- Enfermedad renal crónica

- Inmunosupresión

- Tabaquismo pesado y exposición al humo de biomasa

Los estudios han demostrado que la mucosa nasal, las conjuntivas y, con menos frecuencia, la boca son los portales de entrada para los virus respiratorios. La transmisión del coronavirus SARS-CoV-2 puede ocurrir por vía aérea a través de las gotículas de saliva que transportan patógenos infecciosos y se emiten al hablar, toser o estornudar, pero también al estrechar la mano de una persona enferma o tocar una superficie contaminada. Estas gotículas respiratorias pueden viajar y sobrevivir en superficies horizontales cercanas al paciente. Recientemente se ha descrito que la vía fecal-oral es otra posible fuente de transmisión.

El personal de la salud puede tener contacto directo con las manos sobre estas superficies, que contienen secreciones respiratorias infecciosas y autoinocularse con el patógeno después de manipular sus ojos, mucosa nasal, boca y piel facial no intacta.

La propagación nosocomial del virus ha sido bien documentada y parece agudizar la epidemia en algunos lugares. Dentro de las primeras 6 semanas de epidemia en China, se confirmaron 1716 casos entre los trabajadores de la salud, y al menos 5 murieron $(0,3 \%)$. Aunque las medidas apropiadas para el control de infecciones hospitalarias pueden prevenir la transmisión nosocomial del SARS-CoV-2, trabajar en un ambiente de alto riesgo, más horas de servicio y tener una higiene de manos subóptima después del contacto con los pacientes son factores que se asocian con mayor riesgo de infección. A principios de marzo de 2020, cerca de la mitad de los 200 casos reportados en Cerdeña se encontraban en el hospital y entre otros trabajadores de la salud. A finales de marzo de 2020, el personal médico representaba el 12\% y el $8 \%$ de los infectados en España e Italia, respectivamente. Hasta el 28 de marzo, habían fallecido 51 médicos en Italia y 5 en Francia.

Como ya se mencionó, debido a la actual epidemia por el virus SARS CoV-2/COVID-19, el personal de salud es el más expuesto al contagio con este virus. Por lo anterior, la OMS estableció unas directrices estándar, que fueron consideradas en las recomendaciones del Consenso Colombiano de Atención, Diagnóstico y Manejo de la Infección por SARS-CoV-2/COVID-19 en establecimientos de atención de salud, para minimizar así el riesgo biológico en los trabajadores que atienden pacientes con sospecha de infección o infección por SARS-CoV-2/COVID-19, las cuales incluyen el uso de:

- Protección ocular

- $\quad$ Tapabocas o máscaras N95

- Caretas

- Gorros

- Guantes

- $\quad$ Batas o trajes especiales

- Lavado frecuente de manos

- Higienización de manos

Todos estos elementos de protección que utiliza el personal de la salud para disminuir el riesgo de infección por SARS-CoV-2/COVID-19, usados durante largas jornadas laborales, y la higiene personal excesiva pueden alterar la barrera cutánea y de las mucosas, causando lesiones por presión y fricción. El sitio más afectado es el puente nasal ( $83 \%$, debido al uso de gafas protectoras), y se ha descrito: dermatitis de contacto, prurito, urticaria por presión y exacerbación de procesos inflamatorios preexistentes, como dermatitis seborreica y acné. Los signos clínicos más frecuentes son: eritema, pápulas, maceración y descamación, además de síntomas como ardor, prurito y escozor. Todos estos hallazgos se relacionaron con el uso de EPP en el 97\% de los individuos de una población de 542 trabajadores de atención médica de primera línea.

A continuación, se presentan las alteraciones mejor descritas. 


\section{DERMATITIS DE CONTACTO}

La dermatitis de las manos es un trastorno cutáneo común, con una prevalencia anual de aproximadamente el 10\% entre la población general. Es una enfermedad crónica con un impacto negativo significativo y duradero en la calidad de vida (QoL) de quienes la padecen. Más de la mitad de todos los pacientes con dermatitis de las manos están colonizados por bacterias (Staphylococcus aureus), y el riesgo de colonización está fuertemente relacionado con la gravedad de la dermatosis. Cuando el personal de salud sufre la enfermedad, puede convertirse en transmisor de bacterias potencialmente peligrosas para los pacientes que tiene a su cuidado, cuya inmunidad puede estar disminuida.

La dermatitis de contacto irritativa de las manos usualmente ocurre por el lavado frecuente, la manipulación de detergentes, alcohol, látex, solventes o sustancias con un pH alto, y por el uso de limpiadores enzimáticos que empeoran la humedad y la oclusión. Por otro lado, la dermatitis de contacto alérgica de las manos suele ser desencadenada por la exposición repetitiva o cró- nica a diferentes alérgenos, que pueden ser específicos del trabajo (figura 1).

En los trabajadores de la salud, particularmente en las enfermeras, es más frecuente la dermatitis de contacto irritativa que la alérgica. El látex presente en los guantes es la causa más frecuente de dermatitis de contacto alérgica en esta población. Aproximadamente, el $17 \%$ de los trabajadores de la salud se sensibilizan al látex de los guantes. También ocurren formas mixtas (dermatitis de contacto irritativa y alérgica), en las que intervienen diversos factores endógenos y exógenos. A pesar de lo anterior, el lavado frecuente de las manos y el uso de antisépticos y desinfectantes son medidas indispensables, que han probado servir para la prevención del contagio con SARS-CoV-2/COVID-19, por lo cual se han convertido en prácticas obligatorias durante la pandemia. Todos estos factores contribuyen a la disfunción de la barrera cutánea, pues producen cambios en el pH de la piel, modifican su composición lipídica y favorecen la disbiosis del microbioma cutáneo, lo cual aumenta el riesgo de aparición de dermatitis y sensibilización cutánea a alérgenos, además de la colonización por patógenos.

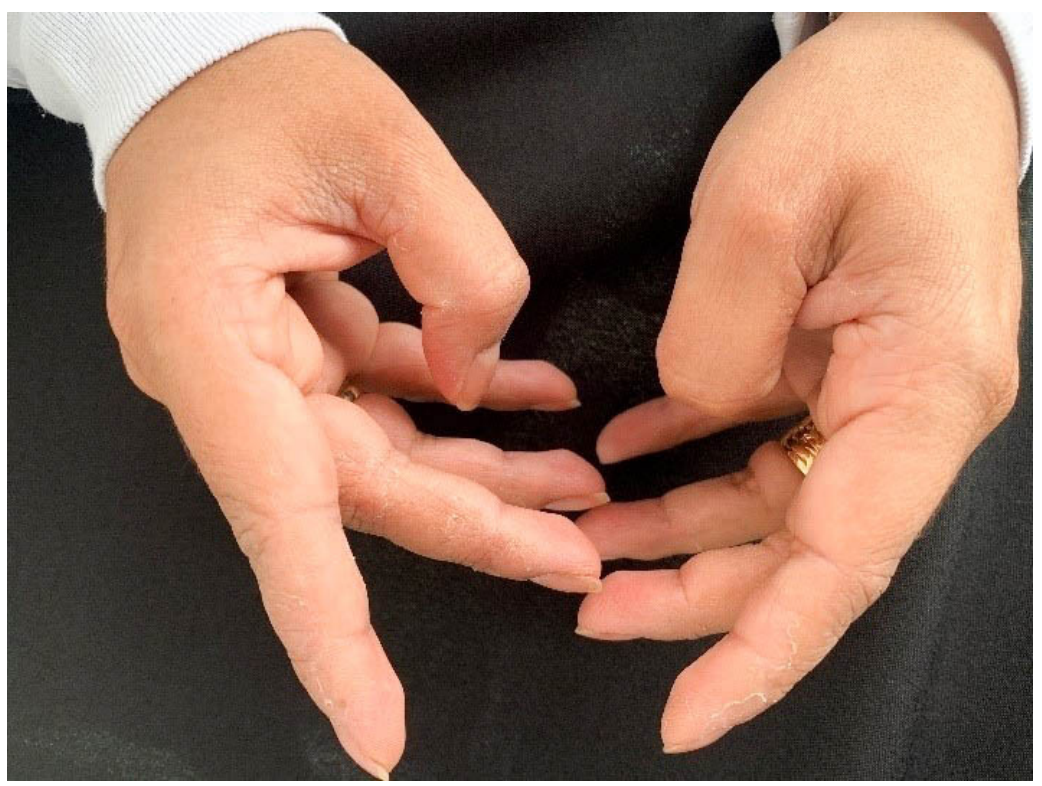

Figura 1. Descamación secundaria al lavado frecuente de las manos en el personal de la salud. 

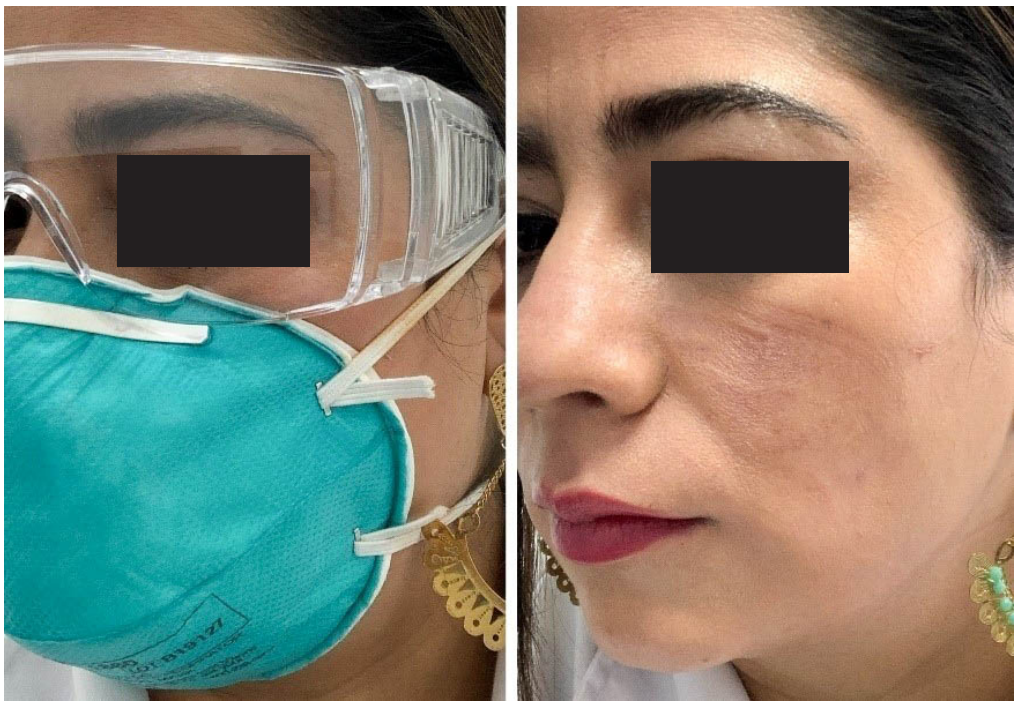

Figura 2. Sitios de presión que siguen la configuración de la máscara N95.
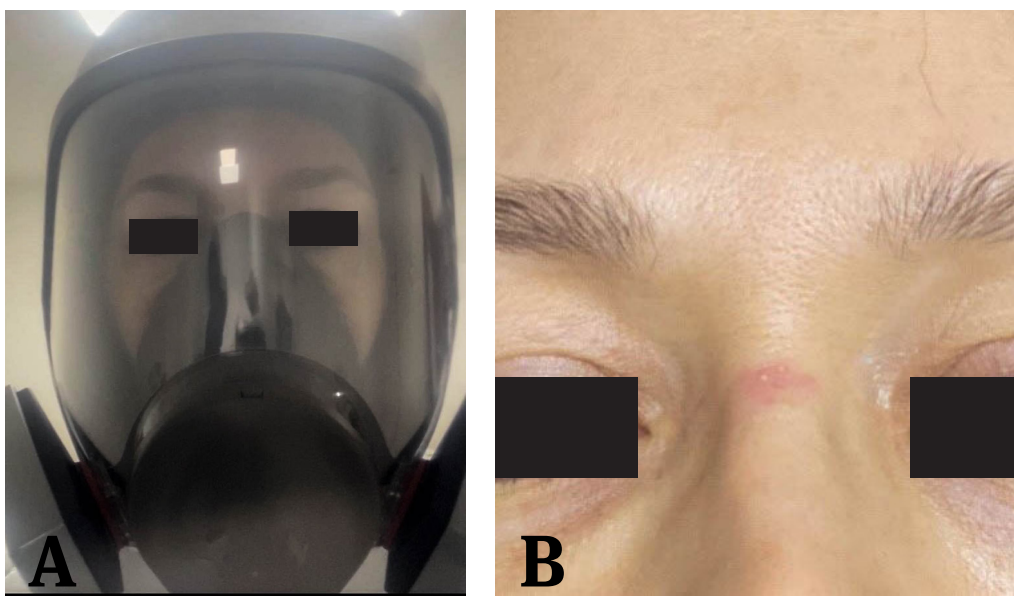

Figura 3. A) Máscara protectora utilizada por el personal de salud en contacto con pacientes hospitalizados en la UCI-COVID. B) Eritema leve en el sitio de contacto con este dispositivo.

\section{LESIONES POR PRESIÓN}

El uso de gafas de seguridad y tapabocas durante un tiempo prolongado genera áreas de presión sobre la piel, que a menudo adopta la misma configuración (forma) del dispositivo (figura 2). Inicialmente se evidencia eritema, edema leve y acentuación de los pliegues de contacto con los dispositivos, que pueden durar minutos o incluso horas (figura 3). Dependiendo de la intensidad, se pueden formar úlceras después de
2 a 6 horas de presión continua, las cuales se definen como la pérdida de continuidad de la superficie cutánea secundaria a una presión repetida aplicada durante largos períodos sobre la piel, los tejidos blandos, los músculos y los huesos, donde la presión externa excede la presión de cierre capilar. Se ha evaluado el efecto protector de los apósitos sobre el desarrollo de úlceras por presión y se ha sugerido como acción preventiva el uso de apósitos, por su capacidad para reducir/disipar la presión y las cargas de corte; aunque 


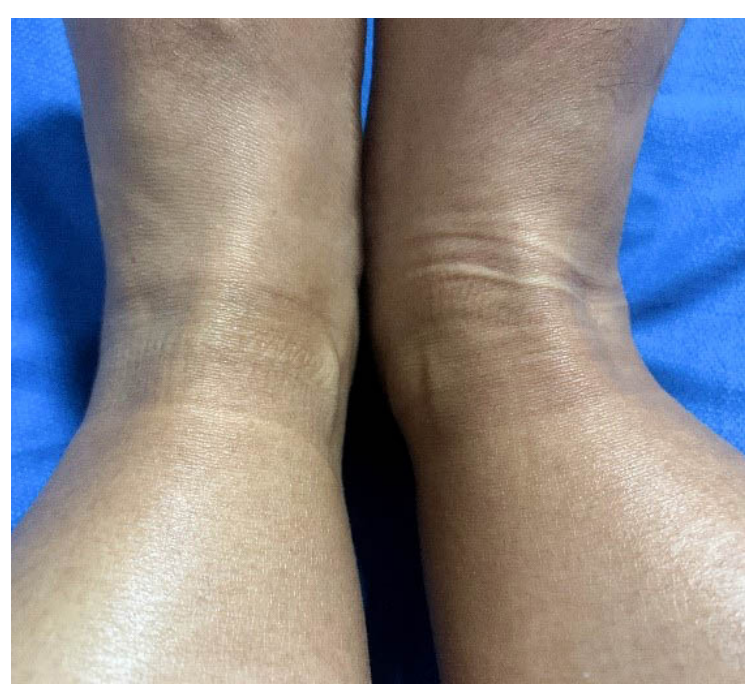

Figura 4.Presión de las medias en el cuello del pie luego de un turno de 12 horas en la UCI-COVID, donde el personal de la salud debe utilizar EPP especial.

no todos los apósitos proporcionan el mismo nivel de protección, los apósitos de varias capas parecen redistribuir las fuerzas mejor que los apósitos monocapa.

La urticaria por presión es otra dermatosis que puede presentarse secundaria al uso de los EPP. Se caracteriza por sensación de incomodidad, eritema y edema facial, con prurito como síntoma predominante (figura 4).

\section{XEROSIS Y DESCAMACIÓN}

Como resultado del lavado excesivo de la piel, el proceso de recambio epidérmico se acelera, de tal forma que se evidencia una descamación sutil, o incluso muy marcada, que le confiere a la piel un aspecto craquelado, con sensación áspera y síntomas como escozor o prurito (figura 5).

\section{ACNÉ}

El acné es una dermatosis inflamatoria multifactorial que afecta la unidad pilosebácea, en donde el efecto oclusivo y la disbiosis del microbioma influyen en la fisiopatogenia de la enfermedad. Las lesiones de acné pueden manifestarse como comedones abiertos y cerrados, pápulas inflamatorias, pústulas y nódulos. La cara, el pecho y la espalda superior son las localiza-

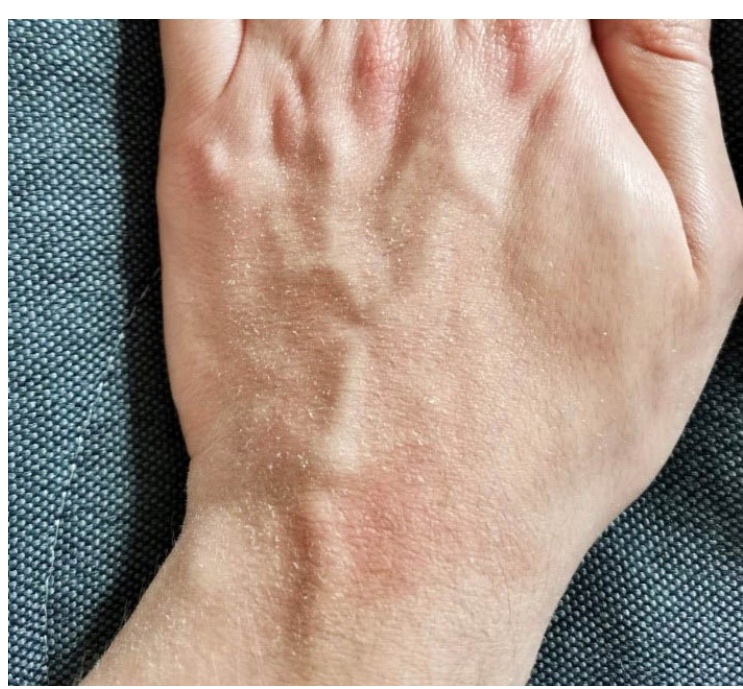

Figura 5. Xerosis con descamación superficial secundaria al lavado frecuente de las manos.

ciones más frecuentes. El uso continuo de EPP puede causar acné debido a la oclusión, que se exacerba con la fricción y el lavado continuo de las zonas afectadas.

\section{HIPERHIDROSIS}

La ropa de protección personal (RPP) es un componente importante de la seguridad de los trabajadores. Si bien la función principal de la RPP es proteger al usuario de los riesgos externos del lugar de trabajo, la carga térmica impuesta por la RPP, particularmente en ambientes cálidos y húmedos, puede afectar negativamente la salud de los trabajadores y su eficiente desempeño. Cuando se realizan tareas extenuantes o durante jornadas prolongadas, en condiciones ambientales calurosas, la producción de sudor aumenta y puede exacerbarse cuando la RPP genera una barrera para la disipación de calor por evaporación. La temperatura del cuerpo humano está regulada por el intercambio de calor entre el cuerpo y el entorno térmico. Cuando este mecanismo no puede compensar el aumento de calor, la temperatura central puede aumentar, causando cambios fisiológicos que incrementan la sudoración y predisponen a humedad y maceración, factores de riesgo para la colonización bacteriana y por hongos. Asimismo, la RPP favorece la aparición de miliaria, una erupción que en algunos casos puede ser sintomática y causar prurito. 


\section{ALTERACIONES EN EL PELO Y LAS UÑAS}

La dermatitis seborreica del cuero cabelludo se exacerba ante condiciones de estrés. El uso continuo de gorros de protección aumenta el calor local y la humedad, empeorando la descamación, la formación de costras y el prurito. El estrés también induce y exacerba la caída masiva de cabello (efluvio telógeno) y la alopecia areata. Por otro lado, tal como ocurre con la piel de las manos, el lavado excesivo afecta las uñas, que pueden sufrir alteraciones como fragilidad de las láminas, desprendimiento por capas (onicosquisis) (figura 6), cambios en el color, e incluso cambios periungueales, que son potenciales puertas de entrada a microorganismos, agravando así cuadros de paroniquia y dermatitis de las manos.

\section{EFECTOS DEL ESTRÉS SOBRE DERMATOSIS PREVIAS}

Las dermatosis inflamatorias crónicas como la dermatitis seborreica, la dermatitis atópica, la psoriasis, el vitiligo y el acné se suelen exacerbar ante condiciones de estrés. La pandemia actual es un evento estresante, factor de riesgo para desencadenar o agravar estas dermatosis en trabajadores de la salud.
Aunque la conjuntivitis folicular y la anosmia no son manifestaciones secundarias al uso de EPP, los trabajadores de la salud deben tener en cuenta que este virus afecta la mucosa conjuntival y la mucosa nasal.

\section{Conjuntivitis folicular}

El virus afecta las conjuntivas tarsal y bulbar, y puede generar procesos inflamatorios como conjuntivitis folicular y quemosis, la cual es indistinguible de otras conjuntivitis virales. Por esta razón, se debe sospechar infección por SARS-CoV-2/COVID-19 en personal de la salud que presente conjuntivitis, asociada a fiebre, tos o dificultad respiratoria. Asimismo, se ha evidenciado ARN del SARS-CoV-2/COVID-19 en las lágrimas, por lo cual se recomienda la desinfección en caso de entrar en contacto con estas secreciones.

\section{Anosmia y disgeusia}

Cerca del 80\% de los pacientes con infección por el SARS-CoV-2/COVID-19 sufren pérdida total del olfato, y el $88 \%$ tiene dificultades para identificar los sabores dulce, salado o amargo. También se ha informado que el 79\% no presenta síntomas nasales, como obstrucción o rinorrea, que comúnmente se asocian a la pérdida del olfato y el gusto, como ocurre en el resfriado común. Por tal motivo, estas alteraciones deben ser reconocidas como síntomas que obligan a sospechar infección por SARS-CoV-2/COVID-19 en el personal de

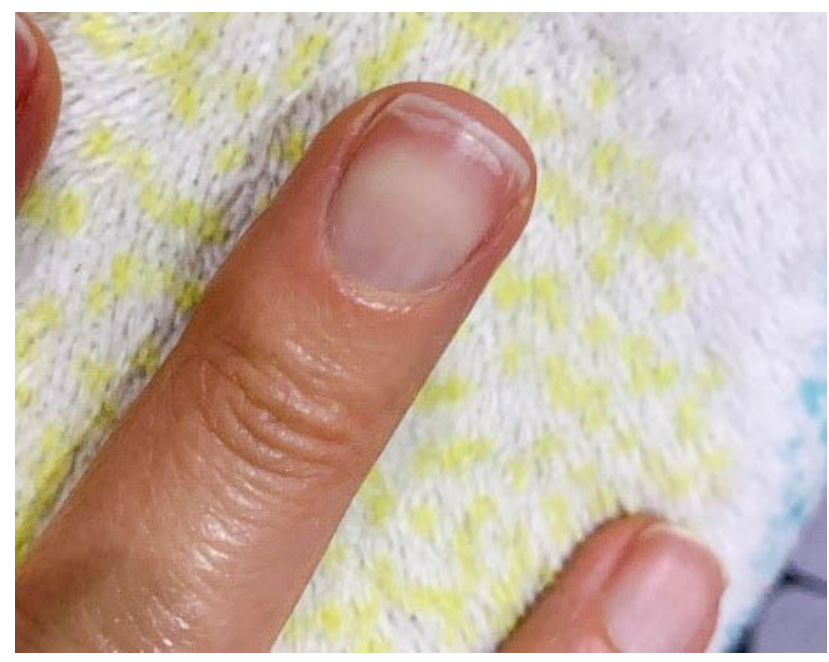

Figura 6. Desprendimiento en capas de las uñas (onicosquisis) causado por el lavado frecuente de las manos. 
la salud que los refiera. La anosmia es un hallazgo útil para la detección temprana de la enfermedad, pues en el $12 \%$ de los pacientes la pérdida total del olfato es el primer síntoma, asociado a fiebre, tos o dificultad respiratoria.

Por las razones expuestas anteriormente, como representantes de la Asociación Colombiana de Dermatología y Cirugía Dermatológica (AsoColDerma) y el Hospital Universitario Centro Dermatológico Federico Lleras Acosta, como ente asesor del Gobierno colombiano, nos permitimos hacer las siguientes recomendaciones encaminadas a reducir el riesgo de lesiones cutáneas en el personal de la salud durante la presente pandemia, derivadas del uso de los implementos de protección personal, indispensables para la prevención del contagio con SARS-CoV-2/ COVID-19.

Estas recomendaciones son de orientación general para la práctica clínica, y el personal de la salud las debe seguir de forma individualizada, de acuerdo con sus necesidades y con los recursos disponibles.

\section{RECOMENDACIONES}

\section{GENERALES PARA LA HIGIENE E HIDRATACIÓN DE LAS MANOS Y EL ROSTRO}

La higiene de manos se puede realizar de dos formas:

1. Lavado de manos y rostro: lávese las manos con agua y jabón. Se recomienda el lavado de manos durante al menos 20 segundos con jabones antisépticos o neutros. Las personas con factores de riesgo (antecedentes de dermatitis atópica, dermatitis de contacto o procesos inflamatorios de la piel) deben utilizar surfactantes sintéticos (syndet, por sus siglas en inglés) o aceites de ducha, como sustitutos del jabón. Aunque no existen estudios que permitan determinar el efecto de los syndet sobre el coronavirus, los tensoactivos presentes en estos productos tienen la capacidad de inducir la disrupción lipídica de la cubierta viral; por lo tanto, serían efectivos.

2. Higienización de las manos: si no hay agua y jabón disponibles, use soluciones hidroalcohólicas, idealmente un desinfectante para manos a base de alcohol, por lo menos al 70\%.
- El lavado de manos con jabón se recomienda antes, durante y después de la jornada laboral, cuando entre en contacto con superficies o con fluidos contaminados, antes de comer, y antes y después de ir al baño. El resto de higiene se puede realizar con soluciones hidroalcohólicas.

- El lavado de manos en la jornada laboral se debe realizar siguiendo los 5 momentos descritos por la OMS.

- Para el lavado del rostro, se recomienda utilizar agua y jabón con $\mathrm{pH}$ neutro, antes y después de la jornada laboral. El personal con factores de riesgo (antecedente de dermatitis atópica, dermatitis de contacto o procesos inflamatorios de la piel) debe utilizar sustitutos del jabón, como los syndet.

- Si tiene dermatitis o algún otro proceso inflamatorio cutáneo, no use soluciones hidroalcohólicas; un correcto lavado de manos es suficiente para eliminar el SARS-CoV-2/COVID-19.

- Evite lavarse las manos y el rostro con agua caliente, esto podría exacerbar síntomas como el prurito y el ardor.

Hidratación de la piel:

- $\quad$ Se recomienda aplicar humectantes o emolientes después de cada lavado de manos y cuantas veces sea necesario.

- En aquellos pacientes con alteración de la barrera cutánea, se recomienda utilizar emolientes o humectantes libres de fragancias, colorantes y con preservantes hipoalergénicos, cuyo mayor contenido sean las ceramidas. No olvide aplicar estos productos en las orejas.

- Después del lavado del rostro, se recomienda utilizar cremas hidratantes libres de fragancias, colorantes y con preservantes hipoalergénicos. Humecte la piel antes del uso de gafas, máscaras y tapabocas, idealmente con cremas cuyo mayor contenido sean ácidos grasos hiperoxigenados.

\section{RECOMENDACIONES DEL} CUIDADO DE LA PIEL FRENTE AL USO DE ELEMENTOS DE
PROTECCIÓN PERSONAL

1. Uso de guantes:

- $\quad$ Evite el uso de guantes durante períodos prolon- 
gados. Se recomienda aplicar emolientes o humectantes antes y después del contacto con los guantes. En lo posible, utilizar guantes de látex, $\mathrm{y}$ en aquellos pacientes con factores de riesgo (antecedente de dermatitis atópica, dermatitis de contacto o procesos inflamatorios de la piel) usar guantes de nitrilo, ya que los guantes de vinilo son permeables. Utilice idealmente guantes sin talcos, para minimizar la formación de residuos.

- El uso de doble guante se recomienda en las situaciones especiales que lo requieran, de acuerdo con las recomendaciones de la OMS.

- No se recomienda la higiene sobre los guantes, ya que podría crear un residuo que genera contaminación. Siempre se debe realizar la higiene sobre la piel.

- Cambie los guantes con frecuencia, estos son porosos y pierden la impermeabilidad después de 20 a 30 minutos de uso.

- $\quad$ El uso continuo y prolongado de guantes, y la falta de recambio, causa oclusión y un estado de hiperhidratación de la epidermis, que es clínicamente observable como maceración y erosión, lo cual podría favorecer el desarrollo de una dermatitis de contacto y la colonización de la piel por bacterias y hongos.

- Al usar guantes, cerciórese de que se han eliminado los residuos de jabón de la superficie de las manos, ya que el efecto oclusivo que produce el material del guante puede potenciar los efectos irritantes o alergénicos de los jabones.

- En pacientes con erosiones por maceración, se pueden usar compresas hidropáticas con ácido bórico al 3\%, solución salina normal o protectores cutáneos con óxido de zinc al 20\%.

- $\quad$ Elimine o sustituya las actividades que son perjudiciales para la piel. Las sustancias peligrosas deben eliminarse, en la medida de lo posible, o reemplazarse por sustancias, preparaciones o productos que no lo sean o que sean menos peligrosos para la salud y la seguridad.

- Ante la presencia de dermatitis de contacto, cuya única manifestación sea eritema, será suficiente el uso de corticoides de baja potencia, como la hidrocortisona al 1\% o la desonida al 0,05\%, combinados con cremas humectantes, dos veces al día. En caso de persistir la molestia o de mayor severidad, se recomienda consultar al dermatólogo.

1. Uso de gafas de protección, máscaras de protección y tapabocas:
- $\quad$ Recuerde que las gafas pueden generar trauma por presión, urticaria por presión, dermatitis de contacto o empeoramiento de las enfermedades dermatológicas previas.

- Al usar gafas de protección, estas deben cubrir la totalidad de sus ojos y la piel periocular. La protección puede incluir un protector facial que cubra completamente la frente y los lados de la cara.

- Use las gafas a su medida, sin ajustarlas de forma excesiva, e intente apoyarlas en sitios diferentes cada vez que las utilice.

- Cubra las superficies de contacto permanente (puente nasal y pómulos) con elementos que disminuyan la presión que se genera sobre la piel, tales como: apósitos de película semipermeables (una membrana delgada de poliuretano recubierta con una capa de acrílico adhesivo) o apósitos hidrocoloides (un apósito que contiene una dispersión de gelatina, pectina y carboximetilcelulosa junto con otros polímeros y adhesivos que forman un dispositivo flexible). Aunque no regulen completamente la humedad ni la temperatura, ya que son oclusivos, también se pueden utilizar apósitos de espuma (una lámina de espuma de poliuretano, hidrófoba) o apósitos finos, preferiblemente con silicona.

- Establezca y garantice períodos de descanso de duración no inferior a 15 minutos, en los que se interrumpa la presión, cada 4 a 6 horas.

- Se recomienda evitar el uso de tapabocas N95 durante más de 8 horas continuas.

- $\quad$ Si sufre de urticaria por presión, se recomienda el uso de antihistamínicos para prevenir los episodios, además de las medidas ya mencionadas para disminuir la presión.

- El tapabocas resortado puede ocasionar presión y dolor en la piel de la región retroauricular; en tal caso, considere usar tapabocas de amarre de tiras, ajustándolo adecuadamente.

\section{RECOMENDACIONES PARA EL CUIDADO DE LAS MUCOSAS}

- $\quad$ No toque sus ojos, nariz o boca, a menos que previamente se realice la higiene de manos.

- En caso de sospechar exposición a secreciones o aerosoles en la zona periocular, debe lavar con agua y jabón, cerrando fuertemente los párpados, ya que el contagio del SARS-CoV-2/COVID-19 puede ocurrir a partir del paso del virus desde la piel hacia la mucosa. 
- Tenga precaución y evite el contacto directo con los ojos con soluciones hidroalcohólicas.

- No existen medicamentos en colirio que sirvan para tratar la conjuntivitis de origen viral, a diferencia de la bacteriana o la alérgica. Su uso debe ser autorizado por personal calificado, preferiblemente un oftalmólogo.

- $\quad$ Si es usuario de lentes de contacto, considere el cambio a gafas, con el fin de evitar el contacto y manipulación de la conjuntiva. Las gafas proporcionan una barrera adicional de protección.

- En caso de no poder omitir el uso de lentes de contacto, procure utilizar lentes de contacto desechables, de reemplazo diario. Esta recomendación no modifica el uso de gafas o máscaras protectoras.

- Tenga en cuenta que debe cumplir las indicaciones de lavado de manos de la OMS antes y después de la manipulación, inserción y extracción de los lentes, así como el protocolo de limpieza y desinfección establecido para sus lentes y el estuche donde los almacena.

- La mucosa nasal expresa el receptor de la enzima convertidora de angiotensina 2 (ECA-2); por lo tanto, es una potencial puerta de entrada para el virus. El personal de la salud debe limpiarse las fosas nasales antes de iniciar su jornada laboral y previo uso de los EPP. Puede utilizar un aspirador nasal o solución salina al o,9\%.

- $\quad$ Si tiene sequedad de la mucosa nasal, puede usar lubricantes nasales en gel o en espray, antes y después de usar los EPP, previo lavado de manos, siguiendo siempre las recomendaciones de la OMS.

- $\quad$ Si la mucosa nasal entra en contacto con fluidos de pacientes con infección por SARS-CoV-2/ COVID-19, se deben limpiar las fosas nasales y el vestíbulo nasal con un algodón impregnado en alcohol, en una concentración del 70\% como mínimo.

- Los vellos nasales pueden ser un medio que facilite la adhesión viral; por lo tanto, se deben recortar.

- Idealmente, el personal de la salud no debe tener barba ni bigote, pues podrían facilitar la infección.

- Lávese los dientes en la mañana, al terminar la jornada y en la noche.

- Nunca se lave los dientes en medio del ambiente hospitalario.

- Aplique bálsamo labial antes de usar los EPP (prefiera productos sin saborizantes, fragancias o colorantes, y con preservantes hipoalergénicos).

\section{OTRAS RECOMENDACIONES}

\section{PARA TENER EN CUENTA}

- El efecto oclusivo de los gorros protectores puede aumentar la absorción de contactantes o medicamentos; también puede provocar prurito y foliculitis, o exacerbar la dermatitis seborreica.

- Cierre con fuerza los labios y los párpados durante el lavado de su rostro y su cabello.

- Las consecuencias de la sudoración que genera el uso continuo de los EPP se pueden prevenir tomando una ducha con agua y jabón después de abandonar las áreas de trabajo contaminadas, y aplicando posteriormente un emoliente o humectante libre de fragancias.

- En caso de hiperhidrosis axilar o en los pies, aplique lociones con cloruro de aluminio del 10\% al 12\%, dos veces al día.

- En pliegues cutáneos aplique protectores cutáneos que contengan óxido de zinc.

- Se recomienda el uso de uniformes de tela ligera, cómodos, y ropa interior de algodón. Se deben cambiar regularmente y cuando están húmedos, para reducir así el efecto de oclusión.

- Para el personal de la salud que utilice trajes especiales durante largas jornadas, se recomienda el uso de ropa interior y medias cómodas, que no queden ajustadas, para disminuir el riesgo de urticaria por presión.

- Mantenga el cabello corto. En caso de usarlo largo, deberá llevarse recogido. En ambos casos siempre se deberá utilizar gorro.

- Al lavarse el cabello, hágalo de forma separada, inclinando la cabeza hacia atrás, evitando que el agua contaminada entre en contacto con su rostro. Hágalo con agua fría, esparciendo el champú con la yema de los dedos y no con las uñas.

- Mantenga las uñas cortas.

- Evite el uso de esmalte. Asimismo, evite portar accesorios (reloj, pulseras, anillos, cadenas, aretes, entre otros) durante la atención médica, ya que el virus podría permanecer en las superficies de estos hasta por 2 días.

- En caso de presentar alguna lesión cutánea secundaria al uso de EPP, consulte al dermatólogo para un diagnóstico oportuno y un tratamiento adecuado. 


\section{Afiliciación de autores:}

1. Especialista en dermatología. Especialista en docencia universitaria, Universidad Militar Nueva Granada. Docente de posgrado, Universidad Militar Nueva Granada. Docente de posgrado, Fundación Universitaria Sanitas. Docente de pregrado, Universidad del Rosario. Vicepresidente, Asociación Colombiana de Dermatología. ORCID http://orcid.org/oooo-0003-3689-5355

2. Especialista en oftalmología, Universidad del Rosario. Especialista en segmento anterior, Universidad Autónoma de Bucaramanga. SO Servicios Médicos y Oftalmológicos S.A.S. ORCID http://orcid.org/oooo-0002-0351-8257

3. Especialista en oftalmología, Universidad Militar Nueva Granada. ORCID http://orcid.org/oooo-0oo1-5594-2183

4. Especialista en otorrinolaringología, Universidad Militar Nueva Granada - Hospital Militar Central. Cirujano plástico facial. Expresidente, Asociación Colombiana de Otorrinolaringología. ORCID http://orcid.org/oooo-0002-9698-3245

5. Oftalmólogo, Fundación Oftalmológica de Santander, Universidad Industrial de Santander. Presidente, Sociedad Colombiana de Oftalmología. ORCID http://orcid.org/oooo-ooo1-6370-7618

6. Especialista en dermatología, Universidad Nacional de Colombia. Fellow de Oncología Dermatológica, Universidad Militar Nueva Granada. ORCID http://orcid.org/oooo-oo02-2301-8348

7. Especialista en dermatología, Pontificia Universidad Javeriana - Centro Dermatológico "Federico Lleras Acosta”. Coordinadora, posgrado de Dermatología, Universidad El Bosque. Jefe, Servicio de Dermatología, Hospital Simón Bolívar. ORCID http://orcid. org/oooo-0002-1924-1256.

8. Especialista en dermatología, Universidad Militar Nueva Granada. Magíster en Epidemiología Clínica, Pontificia Universidad Javeriana. Especialista en docencia universitaria, Universidad Militar Nueva Granada. Jefe, Servicio de Dermatología, Hospital Militar Central. Coordinador, posgrado de Dermatología, Universidad Militar Nueva Granada.

ORCID http://orcid.org/oooo-0002-2476-4991

9. Especialista en dermatología, Universidad Javeriana. Coordinadora, posgrado de Dermatología, Pontificia Universidad Javeriana. Jefe, Unidad de Dermatología, Hospital Universitario San Ignacio. ORCID http://orcid.org/oooo-0002-1125-065X

10. Especialista en dermatología, Fundación Universitaria de Ciencias de la Salud (FUCS). Especialista en dermatología pediátrica, Universitat Autónoma de Barcelona - Hospital de la Santa Creu i Sant Pau. Coordinador, posgrado de Dermatología, Fundación Universitaria de Ciencias de la Salud (FUCS). ORCID http://orcid.org/oooo-00o2-4826-1248

11. Especialista en dermatología, Universidad Nacional de Colombia. Coordinador, posgrado de Dermatología, Universidad Nacional de Colombia. ORCID http://orcid.org/oooo-0oo2-9789-4148

12. Especialista en dermatología, Pontificia Universidad Javeriana - Centro Dermatológico Federico Lleras Acosta. Coordinador, posgrado de Dermatología, Fundación Universitaria Sanitas. ORCID http://orcid.org/oooo-0002-8054-6307

13. Especialista en dermatología, Universidad del Valle. Jefe, Sección de Dermatología y Cirugía Dermatológica, Universidad del Valle. ORCID http://orcid.org/oooo-0003-3582-7748

14. Especialista en dermatología, Universidad del Valle. Jefe, Sección de Dermatología, Universidad Libre de Colombia, Seccional Cali. ORCID http://orcid.org/oooo-0002-3633-7524

15. Especialista en dermatología, Universidad del Valle. Dermatopatología, Universidad de Boston. Jefe, Servicio de Dermatología, Fundación Valle del Lili. Coordinadora, posgrado de Dermatología, Universidad ICESI.

ORCID http://orcid.org/oooo-0002-1962-349X

16. Especialista en dermatología, Universidad Pontificia Bolivariana. Magíster en Epidemiología, Universidad CES. Coordinador, posgrado de Dermatología, Universidad CES. ORCID http://orcid.org/oooo-003-2209-9972

17. Especialista en dermatología, Universidad Pontificia Bolivariana. Coordinador, posgrado de Dermatología, Universidad Pontificia Bolivariana. ORCID http://orcid.org/oooo-0oo1-7225-514X

18. Especialista en dermatología, Universidad de Antioquia. Doctorado, Ciencias Básicas Biomédicas: Énfasis en Inmunología, Universidad de Antioquia. Jefe, Sección de Dermatología, Universidad de Antioquia. ORCID http://orcid.org/oooo-0001-8604-6488

19. Dermatólogo, Pontificia Universidad Javeriana - Centro Dermatológico Federico Lleras Acosta. Coordinador, posgrado de Dermatología, Universidad Autónoma de Bucaramanga.

20. Especialista en dermatología, Hermandad de la Santa Casa de la Misericordia, Sao Paulo. Coordinador, posgrado de Dermatología, Universidad de Cartagena. ORCID http://orcid.org/oooo-0002-8385-6351

21. Especialista en dermatología, Universidad Nacional de Colombia. Coordinador, posgrado de Dermatología, Universidad de Caldas. ORCID http://orcid.org/oooo-0002-0982-4335 


\section{Puntos clave}

- $\quad$ El personal de salud se encuentra en riesgo de infectarse por SARS CoV-2; por lo anterior tenga en cuenta las recomendaciones de la OMS y el Ministerio de salud para minimizar el riesgo de contagio.

- Identifique el peligro, gestione el riesgo y escoja el elemento de protección personal (EPP) adecuado dependiendo de la actividad.

- $\quad$ Para el lavado de manos use jabones con pH neutro o soluciones hidroalcohólicas al 70\%

- Humecte la piel antes y después de usar los EPP con humectantes cuyo mayor contenido sean las ceramidas, libre de fragancias e hipoalergénicos o protectores cutáneos a base de silicona.

- En los sitios de presión por los EPP puede usar apósitos de poliuretano o silicona dándole la forma de como va el EPP.

- Evite tocarse los ojos, la nariz y la boca

- En caso de presentar alguna manifestación cutánea que no mejore con las recomendaciones previamente descritas consulte con el especialista.

\section{AGRADECIMIENTOS}

A los doctores: Óscar Mora Hernández, Clara Inés Ortiz Serrano, Hernán Emilio Duque, Mónica Rivera Jay Lung, Gloria San Clemente, Carlos de La Roche Cadavid, Leonor Molina Prieto, Ricardo Flaminio Rojas, Rafael Humberto Rivera Villamil, Luis Miguel Cobo Segrera, Adriana Arrunátegui Ramírez, Constanza García Paredes (Miembros de la Junta Directiva AsoColDerma 2018-2020). A los doctores Camilo Andrés Morales Cardona y Claudia Marcela Rojas Daza (directora general del Hospital Universitario Centro Dermatológico Federico Lleras Acosta) por el apoyo en la ejecución de este consenso.

\section{LECTURAS RECOMENDADAS}

- Agner T, Elsner P. Hand eczema: epidemiology, prognosis and prevention. J Eur Acad Dermatol Venereol. 2020;34 Suppl 1:4-12. doi: 10.1111/jdv.16061.

- Al Aboud AM, Manna B. Wound pressure injury management. [Updated 2020 Feb 14]. En: StatPearls [Internet]. Treasure Island (FL): StatPearls Publishing; 2020 Jan [citado el 11 de abril de 2020]. Disponible en: https://www.ncbi.nlm.nih.gov/books/NBK532897.

- Alqahtani JS, AlAhmari MD. Evidence based synthesis for prevention of noninvasive ventilation related facial pressure ulcers. Saudi Med J. 2018;39(5):443-52. doi: 10.15537/smj.2018.5.22058.

- Blaak J, Staib P. The relation of $\mathrm{pH}$ and skin cleansing. Curr Probl Dermatol. 2018;54:132-42. doi: 10.1159/000489527.
- Brown BC, Dubrovskiy A, Gvetadze AR, Phalen RN. Chemical permeation of similar disposable nitrile gloves exposed to volatile organic compounds with different polarities Part 1: Product variation. J Occup Environ Hyg. 2020;17(4):165-71. doi: 10.1080/15459624.2020.1721510.

- Cavanagh G, Wambier C. Rational hand hygiene during COVID-19 pandemic. J Am Acad Dermatol. 2020;82(6):e211. doi: 10.1016/j.jaad.2020.03.090.

- Chodosh J, Holland GN, Yeh S. Important coronavirus updates for ophthalmologists [Internet]. 2020 [citado el 6 de abril de 2020]. Disponible en: https://www.aao.org/headline/alert-importantcoronavirus-context.

- Saavedra Trujillo CH. Consenso colombiano de atención, diagnóstico y manejo de la infección por SARS-COV-2/COVID-19 en establecimientos de atención de la salud. Recomendaciones basadas en consenso de expertos e informadas en la evidencia. Infectio. 2020;24(3):1-102. doi: http://dx.doi.org/10.22354/in.v24i3.851.

- Mukamal R, Tuli SS, McKinney JK. Coronavirus Eye Safety [Internet]. American Academy of Ophthalmology. 2020 [citado el 10 de abril de 2020]. Disponible en: https://www.aao.org/eye-health/tips-prevention/ coronavirus-covid19-eye-infection-pinkeye.

- Darlenski R, Tsankov N. Covid-19 pandemic and the skin - What should dermatologists know? Clin Dermatol. 2020. doi: 10.1016/j.clindermatol.2020.03.012.

- Elston DM. Occupational skin disease among healthcare workers during the Coronavirus (COVID-19) epidemic. J Am 
Acad Dermatol. 2020;82(5):1085-6. doi: 10.1016/j.jaad.2020.03.012.

- Foo CCI, Goon AT, Leow YH, Goh CL. Adverse skin reactions to personal protective equipment against severe acute respiratory syndrome - a descriptive study in Singapore. Contact Dermatitis. 2006;55(5):291-4. doi: 10.1111/j.1600-0536.2006.00953.x.

- Grupo Español de Investigación en Dermatitis de Contacto y Alergia Cutánea (GEIDAC). Recomendaciones para minimizar el daño cutáneo derivado del uso de equipos de protección individual frente al SARSCoV-2 [Internet]. 2020 abril 3 [citado el 8 de abril de 2020]. Disponible en: https://bit.ly/3goH8Hh.

- Guan W, Ni Z, Hu Y, Liang W, He J, Liu L, Shan H, Lei CL, Hui DSC, Du B, Li LJ, et al. Clinical characteristics of coronavirus disease 2019 in China. N Eng J Med. 2020;382(18):170820. doi: 10.1056/NEJMoa2002032.

- Organización Mundial de la Salud. Brote de enfermedad por coronavirus (COVID-19): orientaciones para el público [Internet]. Disponible en: https://bit.ly/2Zxkoit.

- Kamps BS, Hoffmann C. COVID Reference [Internet]. 2020 abril 7 [citado el 8 de abril de 2020]. Disponible en: https://covidreference.com/treatment_es.

- Lichterfeld-Kottner A, El Genedy M, Lahmann N, Blume-Peytavi U, Buscher A, Kottner J. Maintaining skin integrity in the aged: A systematic review. Int J Nurs Stud. 2020;103:103509. doi: 10.1016/j.ijnurstu.2019.103509.

- $\quad$ Lin P, Zhu S, Huang Y, Li L, Tao J, Lei T, Song J, Liu D, Chen L, Shi Y, Jiang S, Liu Q, et al. Adverse skin reactions among healthcare workers during the coronavirus disease 2019 outbreak: A survey in Wuhan and its surrounding regions. Br J Dermatol. 2020. abril 7. doi: 10.1111/bjd.19089.

- $\quad$ Marcel-Millet P, Ravier G, Groslambert A. Effect of protective equipment on firefighters' external and internal workloads during a simulated rescue intervention. J Strength Cond Res. 2020. Marzo 12. doi: 10.1519/JSC.0ooooooooooo3551.

- McGoldrick M. Personal protective equipment: protecting the eyes. Home Healthc Now. 2019;37(4):234-5. doi: 10.1097/NHH.0000000000000804.

- Moore ZE, Webster J. Dressings and topical agents for preventing pressure ulcers. Cochrane Database Syst Rev. 2018;12(12):CDoo9362. doi:
10.1002/14651858.CDo09362.pub3.

- Nawrocki S, Cha J. The etiology, diagnosis, and management of hyperhidrosis: A comprehensive review: Therapeutic options. J Am Acad Dermatol. 2019;81(3):669-80. doi: 10.1016/j.jaad.2018.11.066.

- Organización Mundial de la Salud. Medidas de protección básicas contra el nuevo coronavirus [Internet]. 2020 [citado el 6 de abril de 2020]. Disponible en: https://bit.ly/2XwjQ9Z.

- Organización Mundial de la Salud. Seguridad del paciente. Guía para la elaboración a nivel local: Formulaciones recomendadas por la OMS para la desinfección de las manos. Seguridad del paciente [Internet]. 2020. Marzo 30 [citado el 8 de abril de 2020]. Disponible en: https://bit.ly/3gpJBS6 .

- Pärna E, Aluoja A, Kingo K. Quality of life and emotional state in chronic skin disease. Acta Derm Venereol. 2015;95(3):312-6. doi: 10.2340/o0015555-1920.

- Peña-OteroD, Vásquez-DomínguezD, Hernanz-Fernández L, Santano-Magariño A, Jiménez-González V, García-Klepzing JV, Montesinos JVB. Preventing facial pressure ulcers in patients under noninvasive mechanical ventilation: a randomised control trial. J Wound Care. 2017;26(3):128-36. doi: 10.12968/jowc.2017.26.3.128.

- $\quad$ Phalen RN, Dubrovskiy AV, Brown BC, Gvetadze AR, Bustillos M, Ogbonmwan J. Chemical permeation of similar disposable nitrile gloves exposed to volatile organic compounds with different polarities Part 2: Predictive polymer properties. J Occup Environ Hyg. 2020;17(4):172-80. doi: 10.1080/15459624.2020.1721511.

- Sociedad Colombiana de Oftalmología. Recomendaciones y estrategias para prevenir la transmisión del Covid-19 en las unidades de Oftalmología [Internet]. 2020 [citado el 6 de abril de 2020]. Disponible en: https://socoftal.com/covid-19/.

- Sociedad Española de Otorrinolaringología y Cirugía de Cabeza y Cuello. La mayoría de pacientes con COVID-19 presentan alteraciones de gusto y olfato [Internet]. 2020 [citado el 8 de abril de 2020]. Disponible en: https://seorl.net/covid-19-gusto-olfato/.

- Stephens M, Bartley CM. Understanding the association between pressure ulcers and sitting in adults what does it mean for me and my carers? Seating guidelines for people, 
carers and health \& social care professionals. J Tissue Viability. 2018;27(1):59-73. doi: 10.1016/j.jtv.2017.09.004.

- Strauss RM, Gawkrodger DJ. Occupational contact dermatitis in nurses with hand eczema. Contact Dermatitis. 2001;44(5):293-6. doi: 10.1034/j.1600-0536.2001.440508.x.

- WangH, WangS, YuK.COVID-19infectionepidemic: themedicalmanagementstrategiesin Heilongjiang Province, China. Crit Care. 2020;18;24(1):107. doi: 10.1186/s13054-020-2832-8.

- Wang JV, Parish LC. Dermatologic manifestations of the 1918-1919 influenza pandemic. Skinmed. 2019;17(5):296-7.

- Watson C, Troynikov O, Lingard H. Design considerations for low-level risk personal protective clothing:areview.IndHealth.2019;57(3):306-25.doi: 10.2486/indhealth.2018-0040.

- Wu P, Duan F, Luo C, Liu Q, Qu X, Liang L, Wu L. Characteristics of ocular findings of patients with coronavirus disease 2019 (COVID-19) in Hubei Province, China. JAMA Ophthalmol. 2020;138(5):575-8. doi: 10.1001/jamaophthalmol.2020.1291.

- $\quad$ Yan Y, Chen H, Chen L, Cheng B, Diao P, Dong L, Gao X, Gu H, He L, Ji C, Jin H, Lai W, Lei T, et al. Consensus of Chinese experts on protection of skin and mucous membrane barrier for healthcare workers fighting against coronavirus disease 2019. Dermatol Ther. 2020;e13310. doi: 10.1111/dth.13310.

- Zhang WR, Wang K, Yin L, Zhao WF, Xue Q, Peng M, Min BQ, Tian Q, Leng HX, Du JL, Chang H, Yang Y, et al. Mental health and psychosocial problems of medical health workers during the COVID-19 epidemic in China. Psychother Psychosom. 2020; 1-9. doi: 10.1159/000507639.

- $\quad$ Zhou F, Yu T, Du R, Fan G, Liu Y, Liu Z, Xiang J, Wang Y, Song B, Gu X, Guan L, Wei Y, et al. Clinical course and risk factors for mortality of adult inpatients with COVID-19 in Wuhan, China: a retrospective cohort study. Lancet. 2020;395(10229):1054-62. doi: 10.1016/So140-6736(20)30566-3. 